\title{
Title: Correlates of hair cortisol concentrations in disadvantaged young children
}

\author{
Authors and Affiliations: Julian G. Simmons ${ }^{1,2 *}$, Francisco Azpitarte ${ }^{3,4,5}$, Fatou Diallo \\ Roost $^{3}$, Eric Dommers ${ }^{3}$, Nicholas B. Allen ${ }^{6}$, Sophie Havighurst ${ }^{2}$, Nick Haslam ${ }^{1}$ \\ ${ }^{1}$ Melbourne School of Psychological Sciences, The University of Melbourne, Australia. \\ ${ }^{2}$ Melbourne Neuropsychiatry Centre, Department of Psychiatry, The University of \\ Melbourne and Melbourne Health, Parkville, Australia. \\ ${ }^{3}$ Brotherhood of St Laurence, Australia. \\ ${ }^{4}$ Melbourne Institute of Applied Economic and Social Research, The University of \\ Melbourne, Australia. \\ ${ }^{5}$ School of Social Sciences, Loughborough University, United Kingdom. \\ ${ }^{6}$ Department of Psychology, University of Oregon, USA.
}

\author{
*Corresponding author: \\ Julian G. Simmons \\ Melbourne Neuropsychiatry Centre \\ National Neuroscience Facility \\ Level 3, Alan Gilbert Building \\ 161 Barry St, Carlton \\ Vic 3053, Australia \\ jgs@unimelb.edu.au
}

Acknowledgements: We are grateful to the Brotherhood of St Laurence and participating families for all the support provided throughout the project.

Funding: This work was supported by the Melbourne Social Equity Institute and the Melbourne Neuroscience Institute at the University of Melbourne. Francisco Azpitarte acknowledges the support by the Australian Research Council Centre of Excellence for Children and Families over the Life Course (project number CE140100027). The Centre is administered by the Institute for Social Science Research at The University of Queensland,

This is the author manuscript accepted for publication and has undergone full peer review but has not been through the copyediting, typesetting, pagination and proofreading process, which may lead to differences between this version and the Version of Record. Please cite this article as doi: $10.1002 /$ smi.2842

This article is protected by copyright. All rights reserved. 
with nodes at The University of Western Australia, The University of Melbourne and The University of Sydney. The views expressed herein are those of the authors and are not necessarily those of the Australian Research Council. Francisco also acknowledges financial support from the Spanish State Research Agency and the European Regional Development Fund (ECO2016-76506-C4-2-R).

Conflicts of interest: none.

\title{
Title: Correlates of hair cortisol concentrations in disadvantaged young children
}

\begin{abstract}
Children from highly disadvantaged families tend to experience worse health, educational, and job outcomes than less disadvantaged peers. However, the mechanisms underlying these relationships remain to be explicated. In particular, few studies have investigated the relationships between the psychosocial influences that children are exposed to early in life, and longer-term cortisol output. This study aims to contribute to the literature by exploring how disadvantaged young children's experiences of family adversity, and parenting and family functioning, are related to their long-term cortisol levels. A sample of 60 children (26 males, $\mathrm{M}$ age $=4.25$ years, $\mathrm{SD}=1.68)$ and their mothers $(\mathrm{M}$ age $=34.18$ years, $\mathrm{SD}=7.11)$ from a low income population took part in a single assessment. Mothers completed questionnaires on the family environment, parenting practices, and child behavior. Children provided a hair sample for cortisol assay, and anthropometric measures. A parsimonious multivariate regression model (including potential predictors identified by a selection algorithm) was used to investigate the correlates of hair cortisol concentration (HCC) in children. Higher levels of
\end{abstract}


social exclusion, being male, and younger age were each associated with higher HCC. Maternal nurturing and emotion coaching were associated with lower HCC. Findings suggest that chronic stress may underlie relationships between adversity and its long-term effects, and that HCC offers a promising method for examining chronic stress in children and evaluating interventions by which it can be ameliorated.

Keywords: Disadvantage; Hair cortisol; Parenting; Children

\section{Introduction}

Disadvantage during childhood is widely accepted to negatively affect a range of health, educational, and vocational outcomes in adulthood (Lipina \& Colombo, 2009; Shonkoff et al., 2012). Disadvantage during childhood is typically characterized through indices of income-poverty; however, more broadly it is viewed as a multidimensional construct which, besides the economic dimension, also incorporates other domains of welfare such as health, education, and community participation (McLachlan, Gilfillan \& Gordon, 2013). Compared to children from well-off families, children growing up in poverty are more likely to be exposed to physical and psychosocial stressors, including low-income, poor housing, family turmoil, neighborhood violence, and family break-up (Evans \& English, 2002). Yet, little is known about the relative contributions of these factors to children's health, behavioral, and educational outcomes. The effects associated with disadvantage emerge early: exposure to poor environmental conditions during the critically formative early years of life significantly impacts children's social, emotional, neurobiological and cognitive functioning and development (Hackman et al., 2010; Shonkoff et al., 2012). There is evidence that cortisol, and the hypothalamic-pituitary-adrenal axis (HPAA) more broadly, may mediate associations between disadvantage and key outcomes (White et al., 2017). For example, salivary cortisol 
levels mediated associations between the income-to-needs ratio and cognitive ability in a low-income population of children (1 - 4 years old) and their families (Blair et al. 2011). Salivary cortisol is, however, a momentary measure of HPAA function, and the relationships between psychosocial influences on development and long-term cortisol output in early childhood remain to be explicated. In the current study, we aimed to partially address this gap by examining the relationship between a long-term index of child cortisol output with both income-poverty and a multidimensional conceptualization of disadvantage (i.e., economic, health, education and community participation factors), as well as the role of the family environment.

\section{Utility of Hair Cortisol}

Cortisol output has traditionally been assessed in saliva, urine, or serum samples. All these techniques are limited by the highly dynamic nature of cortisol. In particular, factors such as diurnal variation and reactivity to acute stress make it difficult to infer basal levels of cortisol activity from these unstable assessments. Studies are increasingly examining scalp hair, which allows measurement of total systemic cortisol levels over months, as a marker of chronic HPAA and stress (Stalder et al., 2017). Such measurements have shown relationships with the cumulative experience of stressful events in children (Simmons et al., 2016). Hair cortisol concentrations (HCC) have been argued to reflect total free cortisol, and this is supported by a study that found associations between 30 days of multiple salivary collections and the most proximal $1 \mathrm{~cm}$ of hair growth (Short et al., 2016). Importantly, while one recent study found no relationship between brain morphology and HCC (Chen et al., 2016), another study reports that early life adversity (ELA) moderates the relationship between left hippocampal volume and diurnal salivary cortisol levels, such that a relationship was only 
present in children with ELA (Dahmen et al., In Press). This suggests that early environment may be a key factor in explaining relationships with HPAA function; however, few studies have been conducted, to date, on HCC and early environments.

\section{Childhood Disadvantage and Child Hair Cortisol}

Several recent studies have examined relationships between indices related to disadvantage and child HCC. They consistently report a negative association between parental education and HCC (Rippe et al., 2016, Ursache, et al., 2017; Vaghri et al., 2013; Vliegenthart et al., 2016). However, findings for parental income differ, with some finding no association (Ursache, et al., 2017; Vaghri et al., 2013; Vliegenthart et al., 2016), and others a negative association (Rippe et al., 2017). Vliegenthart et al. (2016) also examined neighborhood (postcode) level factors (specifically education, income and employment), and found a negative association with HCC. No study to date has examined relationships between HCC and more specific indices of childhood disadvantage, such as income-poverty (i.e., family income below the poverty line) and social exclusion (i.e., experience of disadvantage in multiple domains, including economic and social), and particularly in a low-income population. A recent review of HCC as a measure of stress in children identified these issues (and others) as important next steps for research in this area (Bates, Salsberry \& Ford, 2017).

\section{Childhood Disadvantage and the Role of Family}

A burgeoning literature has explored the role that family and parental functioning may play in moderating the adverse effects of growing up in disadvantaged environments on a range of health, behavioral, and educational outcomes. While a review of the relevant literature is beyond the scope of this paper, certain key studies are noteworthy. A longitudinal study of 
16,916 children from the Millennium Cohort (from 3 and 7 years of age) modelled the simultaneous effects of neighborhood disadvantage, family poverty, and adverse life events on children's behavioral problems, while exploring the moderating role of parenting (Flouri et al., 2015). They found that all three risk factors predicted childhood problems, but that a positive parent-child relationship buffered risk effects. In related work, the current authors have demonstrated that multidimensional disadvantage, specifically at the neighborhood level, influences brain development through adolescence, but that positive parenting, as Flouri et al. found, moderates this relationship (Whittle et al., 2017). To date, the influence of parenting and family functioning has not been examined in relation to child HCC.

This study aims to address some of the gaps in the literature by exploring how disadvantaged young children's experiences of family adversity, parenting, and family functioning, are related to long-term cortisol levels. We propose and estimate a multivariate regression model for $\mathrm{HCC}$ where potential predictors are selected using an algorithm to enhance the predictive accuracy and power of the statistical model. It was hypothesized that children exposed to:

1. Higher levels of disadvantage, indexed via income-poverty and a multidimensional measure (that includes social and economic dimensions), will display higher levels of $\mathrm{HCC}$; and,

2. Children exposed to higher levels of nurturing parenting/family environments will display lower levels of HCC, consistent with the role of positive parenting as a buffer to adversity in children.

\section{Materials and Methods}

\subsection{Participants}


A sample of 60 mother-child dyads from Melbourne, Australia, were recruited. Children (26 males, $\mathrm{M}$ age $=4.25$ years, $\mathrm{SD}=1.68)$ and their mothers $(\mathrm{M}$ age $=34.18$ years, $\mathrm{SD}=7.11)$ came from families involved in programs run by the Brotherhood of St Laurence (BSL; $n=41$ ), and their social networks $(n=19)$. The BSL is a not-for-profit community organization that works to alleviate poverty in Australia, running programs that target highly disadvantaged communities exposed to multiple socioeconomic stressors. Exclusion criteria included a parent-reported history of developmental or intellectual disorder in the child, and the use of medications that influence cortisol levels. Data were collected during a home visit, which included completion of questionnaires and collection of hair samples. This research was approved by the research ethics committee of the University of Melbourne.

\subsection{Measures}

\subsubsection{Hair cortisol}

Hair was collected from an area approximately $1 \mathrm{~cm}^{2}$, as close to the scalp as possible, from the posterior vertex. The scalp end was marked on samples $>3 \mathrm{~cm}$ in length. Hair samples were sealed in aluminum foil and plastic zipped bags and stored in the dark until assayed. Assays were conducted by Stratech Scientific, where samples were cut down to $3 \mathrm{~cm}$ lengths (from the scalp end) and processed as previously described (Simmons et al., 2016). Hair segments of $3 \mathrm{~cm}$ represent approximately 3 months of hair growth (see Stalder et al., 2017). Quantification was conducted in duplicate using commercial ELISA kits (Salimetrics, USA) according to the manufacturer's instructions. The inter-assay coefficient of variation was $5.9 \%$, and the intra-assay $5.4 \%$. 


\subsubsection{Measures of early adversity}

Family disadvantage was captured using a binary indicator that assesses whether family income was below the Henderson poverty line (HPL), a threshold calculated by the University of Melbourne which is widely used to quantify income-poverty in Australia. For the analysis, the poverty line for the June quarter of 2015 was used (Melbourne Institute, 2015). To control for differences in family size, family incomes were equivalized using the OECD modified equivalence scale that assigns a value of 1 to the first adult, 0.5 to other adults, and 0.3 to any individual below 15 years of age living in the household. To check the robustness of the results to the choice of the poverty line we considered three alternative poverty indicators constructed using income thresholds ranging 95-70\% of the HPL. Furthermore, a continuous measure of family OECD equivalised family income was also considered to capture the income dimension of disadvantage.

We also considered a multidimensional measure of disadvantage that captures both economic and non-economic dimensions of disadvantage. Specifically, we used the social exclusion measure (SEM) developed by the University of Melbourne in cooperation with the BSL (Scutella et al., 2009). Social exclusion has been defined as the "restriction of access to opportunities and [a] limitation of the capabilities required to capitalize on these [opportunities]" (Hayes, Gray, \& Edwards, 2008, p. 6). This measure is regularly used to monitor trends in multidimensional disadvantage in Australia (BSL and Melbourne Institute, 2015) and has been used by, among others, the Australian Government Productivity Commission - a leading national governmental research body - to study socioeconomic disadvantage and microeconomic policy effects in Australia (McLachlan, Gilfillan \& Gordon, 2013). The SEM is a multidimensional composite indicator of disadvantage which 
uses information on 21 zero-one indicators from seven different domains: economic resources; employment; education and skills; health and disability; social; community; and, personal safety ${ }^{1}$. Larger values of the composite measure indicate more disadvantage.

\subsubsection{Family environments and parenting practices}

Family functioning was measured using the relationships component of the Family Environment Scale, which comprises three subscales that quantify the degree of cohesion, expressiveness, and conflict within the family (Moos \& Moos, 1994). Maternal parenting style was assessed using the Parent Behavior Checklist (Fox, 1994), which includes three subscales that measure expectations about the child, discipline and responses to child's behaviors, and the extent of nurturing and support provided to the child; and, the Maternal Emotional Style Questionnaire (Lagace-Seguin \& Coplan, 2005), which assesses the extent of emotion-coaching and emotion-dismissing in responses to children's feelings of sadness and anger. Higher scores indicate more positive parenting and better family functioning.

\subsubsection{Other control variables}

Data on child and family relevant characteristics were also collected for the study. These included child's height, weight, and Body Mass Index $\left(\mathrm{kg} / \mathrm{m}^{2}\right)$; family information data including mother's age, ethnic group, educational level and employment status; family type (lone parent versus couple), number of siblings, and two indicator variables informing whether the principal carer is the biological mother and whether the child's biological father is a member of the household. Child behavior, as an indicator of child functioning, was measured using the parent-report Strengths and Difficulties Questionnaire (Goodman, 1997). We used the one-sided version designed for parents of 4-10 year olds, which includes 25

\footnotetext{
${ }^{1}$ See supplementary A1 and https://www.bsl.org.au/research/social-exclusion-monitor/ for further details.
} 
items to identify emotional, conduct, hyperactivity/inattention, peer relationship problems and pro-social behavior.

\subsection{Statistical analysis}

All data management and analyses were carried out with STATA, version 14. Prior to analyses, all variables were examined for the identification and management of missing and extreme values. Data on weight and height were missing for 24 and 8 children, respectively. These values were imputed using data from the child growth standards published by the World Health Organisation (WHO, 2018), based upon corresponding height/weight norms constructed considering children's gender and age measured in days. Six families in the sample reported being on welfare at the time of the interview but did not provide an estimate of the welfare payments received by the family. Income for those families was imputed using data on the maximum level of welfare payments available to different family types published quarterly by the Melbourne Institute of Applied Economic and Social Research at the University of Melbourne (Melbourne Institute, 2015). HCC of two children were extreme outliers ( $\log \mathrm{HHC}>1.9 \mathrm{pg} / \mathrm{mg}$ ). Within sample information was used to impute the HCC levels of those children. Specifically, the imputed scores were derived using a linear regression model for HCC that included as covariates the child's age, gender, and BMI; mother's age and educational attainment; family type; and the variables capturing the extent of disadvantage at the family, and individual level. Exclusion of those children did not affect main results.

Multivariate linear regression models were used to investigate associations between HCC, adversity and family functioning while controlling for other potential covariates. A $\log _{10}$ transformation was applied to correct the non-normality of HCC. Statistical relationships 
with HCC were estimated using the LARS-OLS hybrid method proposed by Efron et al. (2004). This method encompasses two steps. In the first step, the selection of variables to include in the model is conducted using the least angle regression (LARS) model selection algorithm, which selects a parsimonious set of covariates for the efficient prediction of the endogenous variable. Selection of a parsimonious model is particularly important in contexts where the number of potential predictors is large relative to the sample size, as the use of complex models with a large number of parameters can lead to overfitting problems. Our data included 31 potential covariates for a sample of 60 families, so the use of a selection algorithm is warranted. As shown in Efron et al. (2004), the LARS algorithm generalizes the Lasso and Forward Stagewise selection models widely used in the analysis of highdimensional data. The selection of predictors in the LARS algorithm is based on a sequential process where all regression coefficients are initially set equal to zero and predictors are sequentially added to the model depending on their absolute correlation with the residuals. Once the optimal set of predictors is identified, the second step comprises the estimation of their statistical relationship with HCC using multivariate ordinary least squares (OLS) regression. Initial application of the LARS method provided a set of predictors including 9 of the 31 covariates. This set included the income-poverty indicator, however this variable comprised only six families above the poverty line. To examine the robustness of the LARS results to changes in the threshold for income-poverty, we also considered three alternative poverty indicators constructed using income thresholds ranging $95-70 \%$ of the HPL, that then classified 8, 10, and 12 families as 'non-poor'. (See supplementary A2 for further details on data management and analyses). 


\section{Results}

Table 1 presents the mean and standard deviation of all variables considered in the analysis and their correlation with HCC. We estimated a multivariate linear model which includes the measures of disadvantage, parenting and family environments, and the other controls described in Section 2.2. Application of the LARS selection algorithm, as noted above, did initially include the income-poverty indicator. However, this result was not robust to changes in the threshold for the poverty line (70-95\%), as the income-poverty indicator dropped from the selected set of predictors when the alternative poverty lines were used. To further check the sensitivity of the LARS algorithm to the way income-poverty was measured, we replaced the binary poverty indicator with a continuous measure of family equivalised income. The LARS algorithm, across each of the alternate income variables, yielded a restricted model including the age and gender of the child; dummy variables for couple families, Asian mothers, children living with their biological father; the SEM measure; the FES score for Expression, the PBC score for Nurturing, and the MESQ score for Emotion-coaching. This parsimonious model was then used to estimate relationships with HCC using multivariate OLS regression. (see Supplementary A2 for results of the LARS analyses).

The regression results, as displayed in Table 2 (and significant effects below), demonstrated that there was a positive association between HCC and SEM ( $b=0.23$; $p$-value $=0.043)$, related to hypothesis 1 . Further, that there was a negative association between HCC and PBC (Nurturing; $\quad \mathrm{b}=-0.28 ; \quad \mathrm{p}$-value=0.014) and MESQ (Emotion-coaching; $\mathrm{b}=-0.25 ; \quad \mathrm{p}$ value $=0.028$ ), related to hypothesis 2 . Associations between HCC and covariates were also detected, including a positive association with mother's ethnicity (Asian; $b=0.01 ; \mathrm{p}$ - 
value $=0.017$ ), and negative association with child gender (female; $b=-0.72 ; p$-value $=0.002$ ), and child age $(b=-0.29 ; p$-value $=0.013)$.

\section{[Table 1 here]}

\section{[Table 2 here]}

\section{Discussion}

Our results show that children's HCC levels were significantly associated with a measure of disadvantage, two parenting measures, and several child and family sociodemographic characteristics. HCC was, as hypothesized, higher amongst those exposed to more disadvantage. Amongst the measures of family disadvantage considered in the analysis, while initial modelling indicated that income-poverty demonstrated a relationship with HCC, this result was not robust to changes in the poverty lines used to define income-poverty, likely due to the small number of families living above the poverty line taking part. When alternative definitions of income-poverty and equivalised income were considered, the income measures did not pass the LARS test and the only measure of disadvantage included in the final parsimonious model was the multidimensional social exclusion measure. Estimates from the adjusted model show that children from families with higher levels of social exclusion had higher HCC. HCC was also, as hypothesized, negatively associated with mothers reporting higher levels of nurturing and emotion-coaching parenting styles. Negative associations with HCC were detected for child sex and age, such that being female and older were associated with lower HCC.

No previous study has investigated relationships between social exclusion and HCC. Social exclusion is primarily a multidimensional index of socioeconomic disadvantage designed to capture individuals' capacity to fully participate in society by quantifying their levels of 
deprivation in a range of dimensions, in addition to economic, such as health, employment, and education, in contrast to the widely-used measure of income and rarely examined income-poverty, which primarily index economic resources (Hayes et al., 2008). Although Ursache et al. (2017) and Vaghri et al. (2013) found no relationship between income and HCC, they did find that HCC was related to parental education, which, could arguably be considered a proxy of social exclusion. However, we did not find a relationship between maternal education and HCC in our sample, consistent with Liu et al. (2016), Rippe et al. (2016) and Vliegenthart et al. (2016). The positive relationship found between social exclusion and HCC in the current study suggests that in this primarily poor population $(90 \%$ living below the poverty line), families' experiences of multiple deprivations and reduced capabilities to fully participate in society is associated with neurobiological differences in the long-term HPAA function of their children.

Importantly, mothers' reports of higher nurturing and more emotion coaching of their children were both associated with lower HCC, suggesting the importance of these dimensions for buffering stress in children growing up in disadvantaged environments, and specifically where exposed to social exclusion. To our knowledge, Ouellette et al. (2015) is the only previous study to investigate the influence of parenting using hair cortisol data, although they examined dyadic, mother-daughter relationships. They found that poor quality parenting moderates the strength of mother-child HCC covariation, but had no main effect on child HCC. Our results are consistent with our recent work with adolescents, where warm/positive parenting styles were found to provide a buffering effect to the negative effects of adversity on brain development and school outcome (Whittle et al., 2017). It was conjectured whether stress induced cortisol, which has demonstrated neurotoxicity (Lee et al., 
2002), may underlie the relationships between adversity and altered brain development. This is consistent with a longitudinal study of low income children assessed from ages 2 to 4 years $(\mathrm{N}=201)$, in which exposure to greater levels of family instability and maternal unresponsiveness predicted elevated (and low $)^{2}$ basal salivary cortisol patterns, which were in turn associated with lower child cognitive functioning at age 4 (Suor et al., 2015). Further study is critical to investigate these links via prospective longitudinal studies with measures of HPAA, brain development, and health/functioning outcomes.

HCC also declined with the age of the child, consistent with the age-gradient in early years reported in Karlén et al. (2013), and girls had lower HCC than boys, even after controlling for age, again consistent with other work with children (e.g., Simmons et al., 2016; Rippe et al., 2016).

As noted, the income-poverty indicator, when based on the Henderson poverty line initially passed the LARS selection test; however, this revealed only six non-poor families in the cohort, and thus brings into question the power to examine related effects, and the risk of type-I error. Our examination of the robustness of the relationship, by varying the poverty line threshold (70-95\%), led to income-poverty not being selected by the LARS algorithm. The non-selection of the income-poverty indicator from the set of best predictors could be caused by the reclassification of some income-poor children with high levels of HCC as nonpoor when lower income standards are used to identify income-poor families. The reclassification of those families is hard to justify on economic and welfare grounds, as their

\footnotetext{
${ }^{2}$ Lower cortisol in relation to adversity has been reported to occur as a longer-term result of early elevations in cortisol that lead to systemic down-regulation of the HPAA, i.e., the attenuation hypothesis (Gunnar \& Vazquez, 2001).
} 
incomes are below the threshold used to define poverty in Australia and therefore their socioeconomic well-being is likely to be lower than that of the typical non-poor family. This, in turn, undermines the validity of the income-poverty indicator as measure of disadvantage, as well as its capacity to predict child HCC in our sample. Family equivalised income, a continuous measure of economic (dis)advantage, was also examined, but did not pass the selection test, consistent with the null findings for income and HCC of Ursache et al. (2017) and Vaghri et al. (2013). In contrast, Rippe et al. (2016) did report an association (negative) between income and HCC in a cohort of 2,484 6-year old children, albeit in a predominantly middle-class cohort.

Certain limitations must be considered in the interpretation of these results. First, the sample size was modest and results should be treated with caution, particularly because of the small number of families with incomes above the income-poverty line. Nonetheless, the finding of elevated cortisol among children exposed to higher levels of social exclusion in this lowincome population is indicative of the negative impact of disadvantage on child biology and stress. Second, parenting measures were self-reported and thus present inherent bias in reporting. Further, although the sample was ethnically diverse, numbers in each category were low, and thus the finding that having an Asian mother $(n=6)$ was associated with higher HCC should be treated with caution. Note that the main conclusions regarding the relationship between HCC, disadvantage, and family environments were found to be robust whether relationships were analyzed controlling for that ethnic group or not. The limitations of the use of hair as a sample medium must also be considered: there are indications that extraneous factors, such as intense exercise, frequent hair washing, or hair treatments can affect HCC (Stalder et al., 2017), however while not specifically examined here, the ages of 
children in this study limits the likelihood of these factors playing a substantive role. Finally, interactions between variables were not explored, due to sample size. Future research is required to address these limitations, explore moderation and mediation, and replicate results.

\section{Conclusions}

This study demonstrates clear associations between young children's exposure to disadvantage, and specifically social exclusion, and elevated HCC. Further, nurturing and supportive parenting styles showed negative associations with HCC, associations that have not been shown previously and yet are consistent with a broader literature. Relationships with income-poverty need to be explored with larger, more diverse cohorts. The relationship between social exclusion, family environments, and children's outcomes may be partially mediated by children's exposure to chronic stress. The present study indicates that chronic stress may be a mechanism underlying the relationship between adversity and its long-term effects, particularly when adversity is defined in terms of families' exposure to multiple deprivations that undermine their capacity to participate in society. The further explication of factors influencing HCC suggests this may be a promising method for examining chronic stress, and its moderators, in children and evaluating interventions by which it can be ameliorated.

\section{References}

Bates, R., Salsberry, P., \& Ford, J. (2017). Measuring Stress in Young Children Using Hair Cortisol: The State of the Science. Biological Research for Nursing, 36, 499-510.

Blair, C., Granger, D.A., Willoughby, M., Mills-Koonce, R., Cox, M., Greenberg, M.T., et al. (2011). Salivary cortisol mediates effects of poverty and parenting on executive functions in 
early childhood. Child Development, 82(6), 1970-1984.

Brotherhood of St Laurence and Melbourne Institute (2015). Social Exclusion Monitor Research Bulletin, June 2015. Brotherhood of St Laurence and Melbourne Institute of Applied Economic and Social Research, University of Melbourne, Melbourne.

Chen, R., Muetzel, R. L., Marroun, El, H., Noppe, G., van Rossum, E. F. C., Jaddoe, V. W., et al. (2016). No association between hair cortisol or cortisone and brain morphology in children. Psychoneuroendocrinology, 74, 101-110.

Dahmen, B., Puetz, V.B., Scharke, W., von Polier, G.G., Herpertz-Dahlmann, B., Konrad K. (2018). Effects of early-life adversity on hippocampal structures and associated HPA axis functions. Developmental Neuroscience, 40(1), 13-22.

Efron, B., Hastie, T., Johnstone, I., \& Tibshirani, R. (2004). Least angle regression. The Annals of statistics, 32(2), 407-499.

Evans, G.W. and English, K., 2002. The environment of poverty: Multiple stressor exposure, psychophysiological stress, and socioemotional adjustment. Child development, 73(4), 12381248.

Fox, R. A. (1994). Parent Behavior Checklist. Brandon, VT: Clinical Psychology Publishing Company.

Flouri, E., Midouhas, E., Joshi, H., \& Tzavidis, N. (2015). Emotional and behavioural resilience to multiple risk exposure in early life: the role of parenting. European Child \& Adolescent Psychiatry, 24(7), 745-755.

Goodman, R. (1997). The Strengths and Difficulties Questionnaire: A research note. Journal of Child Psychology and Psychiatry. 38, 581-86.

Gunnar, M.R., Vazquez, D.M. (2001). Low cortisol and a flattening of expected daytime rhythm: Potential indices of risk in human development. Development and Psychopathology, 13, 515-538.

Hackman D. A., Farah M. J., Meaney M. J. (2010). Socioeconomic status and the brain: mechanistic insights from human and animal research. Nat. Rev. Neurosci, 11, 651-659. 
Karlén, J., Frostell, A., Theodorsson, E., Faresjö, T., \& Ludvigsson, J. (2013). Maternal influence on child HPA axis: a prospective study of cortisol levels in hair. Pediatrics, 132(5), e1333-e1340.

Lagace-Seguin, D. G., \& Coplan, R. (2005). Maternal emotional styles and child social adjustment: Assessment, correlates, outcomes and goodness of fit in early childhood. Social Development, 14, 613-636.

Lee, A., Ogle, W. \& Sapolsky, R. (2002). Stress and depression: possible links to neuron death in the hippocampus. Bipolar Disorders 4, 117-128.

Li, L., Power, C., Kelly, S., Kirschbaum, C. and Hertzman, C. (2007). Life-time socioeconomic position and cortisol patterns in mid-life. Psychoneuroendocrinology, 32(7), 824833.

Lipina, S. J., and Colombo, J. A. (2009). Poverty and Brain Development During Childhood: An Approach from Cognitive Psychology and Neuroscience. Washington, DC: American Psychological Association.

Liu, C. H., Snidman, N., Leonard, A., Meyer, J., \& Tronick, E. (2016). Intra-individual stability and developmental change in hair cortisol among postpartum mothers and infants: Implications for understanding chronic stress. Developmental psychobiology, 58(4), 509-18. McLachlan, R., Gilfillan, G. and Gordon, J. (2013). Deep and Persistent Disadvantage in Australia-Productivity Commission Staff Working Paper.

Melbourne Institute (2015). Poverty Lines: Australia, June Quarter 2015, Melbourne Institute of Applied Economic and Social Research, University of Melbourne, Melbourne.

Moos, R.H. \& Moos, B.S. (1994). Family Environment Scale Manual and Sampler Set.

Department of Psychiatry and Behavioral Sciences, Stanford University Medical Centre, Palo Alto, California.

Ouellette, S.J., Russell, E., Kryski, K.R., Sheikh, H.I., Singh, S.M., Koren, G. et al. (2015). Hair cortisol concentrations in higher-and lower-stress mother-daughter dyads: A pilot study of associations and moderators. Developmental psychobiology, 57(5), 519-534. 
Pink, B. (2013) Technical paper: Socio-economic indexes for area (SEIFA) 2011. Cat. No. 2033.0.55.001. Australian Bureau of Statistics.

Rippe, R.C.A., Noppe, G., Windhorst, D.A., Tiemeier, H., van Rossum, E.F.C., Jaddoe, V.W.V., et al. (2016). Splitting hair for cortisol? Associations of socio-economic status, ethnicity, hair color, gender and other child characteristics with hair cortisol and cortisone. Psychoneuroendocrinology, 66, 56-64.

Scutella, R., Wilkins, R. and Kostenko, W. (2009). Estimates of poverty and social exclusion in Australia: A multidimensional approach. Melbourne Institute of Applied Economic and Social Research, No.2009/26), The University of Melbourne.

Shonkoff, J.P., Garner, A.S., Siegel, B.S., Dobbins, M.I., Earls, M.F., McGuinn, L., et al. (2012). The lifelong effects of early childhood adversity and toxic stress. Pediatrics, 129(1), e232-e246.

Short, S. J., Stalder, T., Marceau, K., Entringer, S., Moog, N. K., Shirtcliff, E. A., et al. (2016). Correspondence between hair cortisol concentrations and 30-day integrated daily salivary and weekly urinary cortisol measures. Psychoneuroendocrinology, 71, 12-18. Simmons, J.G., Badcock, P.B., Whittle, S.L., Byrne, M.L., Mundy, L., Patton, G.C., et al. (2016). The lifetime experience of traumatic events is associated with hair cortisol concentrations in community-based children. Psychoneuroendocrinology, 63, 276-281. Stalder, T., Steudte-Schmiedgen, S., Alexander, N., Klucken, T., Vater, A., Wichmann, S., et al. (2017). Stress-related and basic determinants of hair cortisol in humans: A meta-analysis. Psychoneuroendocrinology, 77, 261-274.

Suor, J.H., Sturge-Apple, M.L., Davies, P.T., Cicchetti, D. and Manning, L.G., (2015). Tracing differential pathways of risk: Associations among family adversity, cortisol, and cognitive functioning in childhood. Child development, 86(4), 1142-1158.

Ursache, A., Merz, E.C., Melvin, S., Meyer, J. and Noble, K.G., 2017. Socioeconomic status, hair cortisol and internalizing symptoms in parents and children.

Psychoneuroendocrinology, 78, 142-150. 
Vaghri, Z., Guhn, M., Weinberg, J., Grunau, R. E., Yu, W., \& Hertzman, C. (2013). Hair cortisol reflects socio-economic factors and hair zinc in preschoolers.

Psychoneuroendocrinology, 38(3), 331-340.

Vliegenthart, J., Noppe, G., van Rossum, E.F.C., Koper, J.W., Raat, H., \& van den Akker, E.L.T. (2016). Socioeconomic status in children is associated with hair cortisol levels as a biological measure of chronic stress. Psychoneuroendocrinology, 65, 9-14.

White, L. O., Ising, M., Klitzing, von, K., Sierau, S., Michel, A., Klein, A. M., et al. (2017).

Reduced hair cortisol after maltreatment mediates externalizing symptoms in middle childhood and adolescence. Journal of Child Psychology and Psychiatry, and Allied Disciplines, 58(9), 998-1007.

Whittle, S., Vijayakumar, N., Simmons, J.G., Dennison, M., Schwartz, O., Pantelis, C., et al. (2017). Role of positive parenting in the association between neighbourhood social disadvantage and brain development across adolescence. JAMA psychiatry, 74(8), 824-832. World Health Organization (2018). The WHO Child Growth Standards, Height and weightfor-age tables, available at http://www.who.int/childgrowth/standards/en/ (accessed on the $12^{\text {th }}$ of March, 2018).

Table 1. Sample descriptive statistics

\begin{tabular}{llccc}
\hline & & Mean & St.dev. & $\begin{array}{c}\text { Correlation } \\
\text { with HCC }\end{array}$ \\
\hline Log HCC (pg/mg) & & 0.46 & 0.40 & 1.00 \\
Mother's age (years) & & 34.18 & 7.11 & 0.03 \\
Child's age (months) & & 56.68 & 19.78 & $-0.31^{* * *}$ \\
Child: Female (\%) & & 57.00 & 50.00 & $-0.32^{* *}$ \\
Mother's ethnic group (\%): & Oceanian & 41.67 & 49.72 & -0.06 \\
& European & 28.33 & 45.44 & -0.08 \\
& Middle-East & 15.00 & 36.01 & -0.16
\end{tabular}


Number of siblings

Mother is biological mother: Yes (\%)

Biological father at home: Yes (\%)

Family type (\%): Couple

Lone parent

Multifamily

Other family type

Mother's education (\%): Postgraduate

Bachelor

Diploma

Certificate

Year 12 or less

Mother's employment (\%): Employed

Unemployed

Out of labour force

\section{Disadvantage}

SEIFA index: Disadvantage

Advantage and disadvantage

Economic resources

Education and occupation

Income poor (HPL threshold): Yes (\%)

Equivalised annual family income (\$)

Social Exclusion Measure

SDQ: Conduct problems

Hyperactivity

Peer problems

Emotional

Pro-social behavior

Total score

Family environments and parenting

FES: Cohesion

Expression

Conflict

PBC: Discipline

Expectations

Nurturing

MESQ: Emotion dismissing

Emotion coaching

\begin{tabular}{|c|c|c|}
\hline Mean & St.dev. & $\begin{array}{c}\text { Correlation } \\
\text { with HCC }\end{array}$ \\
\hline 8.33 & 27.87 & $0.30 * *$ \\
\hline 5.00 & 21.98 & 0.18 \\
\hline 1.67 & 12.91 & 0.00 \\
\hline 1.00 & 0.78 & -0.12 \\
\hline 97.00 & 18.00 & 0.10 \\
\hline 72.00 & 45.00 & 0.21 \\
\hline 70.00 & 46.21 & $0.22 *$ \\
\hline 25.00 & 43.67 & $-0.23^{*}$ \\
\hline 3.33 & 18.10 & 0.05 \\
\hline 1.67 & 12.91 & -0.07 \\
\hline 6.67 & 25.15 & 0.14 \\
\hline 30.00 & 46.21 & -0.04 \\
\hline 10.00 & 30.25 & -0.06 \\
\hline 20.00 & 40.34 & 0.04 \\
\hline 33.33 & 47.54 & -0.03 \\
\hline 35.00 & 48.10 & -0.03 \\
\hline 38.33 & 49.03 & -0.13 \\
\hline 26.67 & 44.59 & 0.18 \\
\hline 918.40 & 122.43 & 0.07 \\
\hline 920.22 & 143.7 & 0.08 \\
\hline 924.13 & 113.26 & 0.07 \\
\hline 935.73 & 109.09 & 0.02 \\
\hline 90.00 & 30.00 & $0.25 *$ \\
\hline 25,244 & 16,250 & -0.18 \\
\hline 1.78 & 0.95 & 0.17 \\
\hline 2.22 & 1.71 & -0.13 \\
\hline 4.47 & 2.62 & 0.11 \\
\hline 1.91 & 1.53 & 0.09 \\
\hline 2.22 & 1.85 & 0.03 \\
\hline 7.43 & 1.78 & -0.13 \\
\hline 17.65 & 6.01 & 0.02 \\
\hline 6.84 & 1.78 & -0.06 \\
\hline 5.34 & 1.99 & 0.13 \\
\hline 5.47 & 2.17 & -0.05 \\
\hline-41.11 & 7.28 & -0.07 \\
\hline 43.04 & 11.3 & -0.14 \\
\hline 52.03 & 12.44 & -0.17 \\
\hline-3.54 & 0.64 & -0.09 \\
\hline-3.93 & 0.56 & -0.19 \\
\hline
\end{tabular}


Notes: Ethnic groups defined following the Australian Standard Classification of Cultural and Ethnic Groups (ASCCEG). SDQ=Strengths and Difficulties Questionnaire; FES =Family Environment Scale; PBC=Parent Behavioral Checklist; MESQ=Maternal Emotional Style Questionnaire. The original scores of the FESconflict, MESQ-emotion dismissing, and MESQ-emotion coaching scales were multiplied by minus one so that larger values indicate more positive forms of parenting. ${ }^{*} p<0.10,{ }^{* *} p<0.05,{ }^{* * *} p<0.01$

Table 2. Parsimonious regression model predicting child cortisol

\begin{tabular}{lcccc}
\hline & Coeff. & Std. Error & t-stat. & $95 \%$ confidence interval \\
\hline Child: female $\ddagger$ & $-0.72 * *$ & 0.22 & -3.33 & $(-1.16,-0.29)$ \\
Child: age (months) & $-0.29 *$ & 0.11 & -2.57 & $(-0.52,-0.06)$ \\
Ethnic group: Asian & $0.02^{*}$ & 0.004 & 2.48 & $(0.002,0.02)$ \\
Family: couple & 0.005 & 0.005 & 0.92 & $(-0.01,0.01)$ \\
Biological father at home & -0.04 & 0.49 & -0.09 & $(-1.03,0.94)$ \\
FES: Expression & 0.20 & 0.12 & 1.75 & $(-0.03,0.43)$ \\
PBC: Nurturing & $-0.28^{*}$ & 0.11 & -2.54 & $(-0.50,-0.06)$ \\
MESQ: Emotion coaching & $-0.25^{*}$ & 0.11 & -2.26 & $(-0.48,-0.03)$ \\
Social Exclusion Measure & $0.23^{*}$ & 0.11 & 2.08 & $(0.01,0.46)$ \\
Constant & 0.04 & 0.24 & 0.18 & $(-0.43,0.52)$ \\
R-squared & 0.48 & & &
\end{tabular}

Notes: FES =Family Environment Scale; PBC=Parental Behavioral Checklist; MESQ=Maternal Emotional Style Questionnaire. $\$=-$ negative coefficient means males have higher HCC.

${ }^{+} p<0.10,{ }^{*} p<0.05,{ }^{* *} p<0.01$ 


\section{University Library}

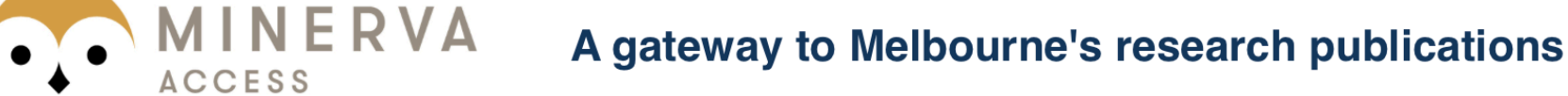

Minerva Access is the Institutional Repository of The University of Melbourne

Author/s:

Simmons, JG;Azpitarte, F;Roost, FD;Dommers, E;Allen, NB;Havighurst, S;Haslam, N

Title:

Correlates of hair cortisol concentrations in disadvantaged young children

Date:

2019-02-01

Citation:

Simmons, J. G., Azpitarte, F., Roost, F. D., Dommers, E., Allen, N. B., Havighurst, S. \& Haslam, N. (2019). Correlates of hair cortisol concentrations in disadvantaged young children. STRESS AND HEALTH, 35 (1), pp.104-111. https://doi.org/10.1002/smi.2842.

Persistent Link:

http://hdl.handle.net/11343/284581 